\title{
A breath test for malignant mesothelioma
}

\section{using an electronic nose}

\author{
Eleanor A. Chapman*, Paul S. Thomas*, Emily Stone ${ }^{\#}$, Craig Lewis ${ }^{\Uparrow}$ and \\ Deborah H. Yates"
}

\begin{abstract}
Malignant mesothelioma (MM) is a rare tumour which is difficult to diagnose in its early stages. Earlier detection of MM could potentially improve survival. Exhaled breath sampling of volatile organic compounds (VOCs) using a carbon polymer array (CPA) electronic nose recognises specific breath profiles characteristic of different diseases, and can distinguish between patients with lung cancer and controls. With $\mathrm{MM}$, the potential confounding effect of other asbestos-related diseases (ARDs) needs to be considered. We hypothesised that as CPA electronic nose would distinguish patients with MM, patients with benign ARDs, and controls with high sensitivity and specificity.
\end{abstract}

$20 \mathrm{MM}, 18 \mathrm{ARD}$ and 42 control subjects participated in a cross-sectional, case-control study. Breath samples were analysed using the Cyranose 320 (Smiths Detection, Pasadena, CA, USA), using canonical discriminant analysis and principal component reduction.

$10 \mathrm{MM}$ subjects created the training set. Smell prints from 10 new MM patients were distinguished from control subjects with an accuracy of $95 \%$. Patients with MM, ARDs and control subjects were correctly identified in $88 \%$ of cases.

Exhaled breath VOC profiling can accurately distinguish between patients with MM, ARDs and controls using a CPA electronic nose. This could eventually translate into a screening tool for high-risk populations.

KEYWORDS: Asbestos, biomarkers, breath testing, electronic nose, mesothelioma, volatile organic compounds

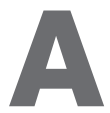
sbestos was extensively used worldwide over the last two centuries and exposure still continues in many countries. Asbestos exposure can lead to one of the more aggressive forms of cancer, malignant mesothelioma (MM), as well as lung cancer [1]. The World Health Organization estimates that globally 90,000 people die from asbestos-related disease (ARD) each year [2]. Asbestos-associated mortality and morbidity have been predicted to rise, and to peak within the next 5 yrs. This is likely to occur even in countries where asbestos usage has been banned because of the long latency period for disease development [3].

The pathophysiological mechanisms that result in the development of malignant disease are rapidly being elucidated [4] due to developments in the understanding of basic mechanisms. Currently, however, there is no method for predicting which asbestos-exposed individuals will develop malignancy [5].

Worldwide, MM causes 15,000-20,000 deaths per year [2]. The prognosis is very poor, with a median survival of only 9 months [6]. Guidelines exist for screening and diagnosis of asbestos-related malignancy, yet in clinical practice diagnostic techniques remain insensitive [7-9]. Conventional techniques for distinguishing between benign and malignant ARD are inaccurate, invasive and difficult for elderly patients [10] who frequently have a high level of intercurrent morbidity. Current treatments are largely ineffective in controlling disease. However, recently several new drugs have become available and trials are now underway to evaluate whether treatment of early disease (including combined surgery and radio-/chemotherapy) will improve survival. Ideally, early detection would allow combination therapy to control or eradicate this neoplasm. A reliable, cheap and noninvasive tool for early diagnosis and/or for screening in high-risk populations is urgently needed.

Because of this, there has been much recent interest in biomarkers for MM, including blood and tissue biomarkers, such as soluble mesothelinrelated peptide, osteopontin and megakaryocyte potentiating factor. However, these are as yet

\section{AFFLLIATIONS}

*Inflammation and Infection Research Centre, School of Medical Sciences, University of New South Wales,

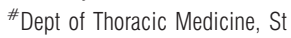
Vincent's Hospital, and "Dept of Medical Oncology, Prince of Wales Hospital, Sydney, Australia.

\section{CORRESPONDENCE}

D.H. Yates

Dept Thoracic Medicine

St Vincent's Hospital

390 Victoria Street

Darlinghurst

NSW 2010

Australia

E-mail: deborahy88@hotmail.com

Received:

March 072011

Accepted after revision:

Nov 232001

First published online:

Dec 192011 
imperfect [11-14]. One novel method of biomarker analysis is through exhaled breath profiling. This has the advantage of being totally noninvasive, quick and very easy for the patient. Breath volatile organic compound (VOC) profiling can distinguish lung cancer patients from healthy controls with a high degree of sensitivity and specificity [5]. More than 4,000 VOCs have been found in exhaled breath, generated mainly from endogenous biochemical pathways including those of lipid peroxidation [15-21]. Techniques used for VOC analysis range from gas chromatography-mass spectrometry and ion mobility spectroscopy to colorimetric and gas sensors. The carbon polymer array (CPA) electronic nose relies on an array of complex nanosensors that produce a breath "smell print", which can be distinguished from other breath patterns using principal component analysis (PCA) [15, 22].

We hypothesised that the CPA electronic nose would detect a breath profile which would accurately distinguish between patients with MM, benign ARDs and healthy control subjects. If this were the case, this could represent a first step towards early detection of MM in asbestos-exposed subjects.

\section{METHODS}

\section{Study design}

This study was approved by the Human Research Ethics Committee of St Vincent's Hospital and the Prince of Wales Hospital (both Sydney, Australia) and subjects gave full written informed consent prior to testing.

The study was of cross-sectional, case-control design in two phases: a training phase and a blinded identification phase. Subjects were assessed once with a standardised questionnaire, spirometry and exhaled breath sampling. The questionnaire included: demographics, occupational history, medical and medication history, smoking history, recent illnesses, and factors known to affect exhaled breath samples in other contexts, e.g. time since last meal, recent alcoholic beverages, smoking and use of mouthwash. Subjects were asked to not eat or drink (excluding water) in the $90 \mathrm{~min}$ prior to testing. To assess reproducibility, five subjects were reassessed three times within 6 months.

\section{Study subjects}

Subjects were recruited from outpatient clinics at St Vincent's Hospital and the Prince of Wales Hospital and controls from community volunteers. Patients with MM and benign ARDs were diagnosed according to current American Thoracic Society (ATS) and European Respiratory Society (ERS) recommendations [7, 23]. MM patients were selected on the basis of immunohistological diagnosis [23] using tissue removed at video-assisted thoracic surgery thoracoscopic biopsy. Patients were not included in the study if there was suspicious cytology and/or clinical and radiological findings without confirmatory histology. Patients with non-malignant ARDs were diagnosed using the following criteria [7, 23]. 1) An occupational history of exposure to asbestos with an appropriate latency period for development of the relevant disease. 2) Compatible clinical findings, e.g. the presence of fine end-inspiratory crackles at the lung bases in the case of asbestosis, diminished overall lung expansion in the case of diffuse pleural thickening, lack of crackles and a normal chest examination in the case of pleural plaques (PPs). 3) The presence of compatible radiological features (PPs, diffuse pleural thickening and lower zone interstitial pulmonary fibrosis). 4) Compatible features on full lung function testing including lung volumes and diffusing capacity of the lung for carbon monoxide. Controls were matched for age, sex and smoking status. Subjects were divided into four groups: 1) MM, 2) asbestosis, 3) pleural disease, including both PPs and diffuse pleural thickening, and 4) control subjects. Nonsmokers were defined as having never smoked cigarettes, and ex-smokers as not having smoked within the last year.

The control group consisted of subjects who reported no asbestos exposure or any lung disease, no current relevant respiratory symptoms and normal spirometry (a pre-bronchodilator forced expiratory volume in $1 \mathrm{~s}(\mathrm{FEV} 1)>80 \%$ predicted and FEV1/forced vital capacity (FVC) $>70 \%$ ). Subjects were selected for smoking status to match smoking history of the two other groups. Exclusion criteria for all groups included a history of recent respiratory tract infection, an acute exacerbation of any underlying respiratory disease in the past 4 weeks and/or other current uncontrolled medical conditions.

\section{Lung function}

All control and ARD subjects underwent spirometry (Autospiro AS-500; Minato Medical Science Company Ltd, Osaka, Japan) according to ATS/ERS guidelines on the same day as breath collection [24]. Most MM subjects experienced significant dyspnoea and were unable to perform repeatable spirometry. FVC (L), FEV1 (L) and vital capacity (VC; L) were measured and the highest value of three manoeuvres was expressed as percentage of the predicted values ( $\%$ pred), calculated using regression equations described elsewhere [25].

\section{CPA electronic nose}

A handheld, portable chemical vapour analyser (Cyranose 320; Smiths Detection, Pasadena, CA, USA) was used to evaluate exhaled breath samples. This device consists of a composite array of 32 organic carbon polymer sensors that respond to gaseous molecules such as VOCs via a change in the electrical resistance of the sensors. The variation of change in resistance of each sensor in response to different breath samples is saved in the inbuilt database. These can then be compared via pattern recognition algorithms to distinguish different smell print patterns.

\section{Exhaled breath collection and sampling}

This involved a training phase and a blinded identification phase conducted on different subjects. In both phases, samples were randomly introduced to the CPA electronic nose system, to prevent bias in smell print generation. In the training phase, breath was sampled from 10 subjects with each condition and 10 controls. In the subsequent validation phase, the exhaled breath of test subjects and controls were randomly introduced into the CPA electronic nose. After $30 \mathrm{~s}$ of sampling, the CPA electronic nose categorised them as either diseased or healthy according to the VOC smell print. To determine baseline drift and variation over time, an assessment of reproducibility was performed on known samples to ensure the results of the training set would remain reproducible.

Breath collection was based on previously validated methods [15]. All subjects sat at rest for $20 \mathrm{~min}$ before sample collection. Subjects first rinsed their mouth with distilled water before 
breathing tidally through a mouthpiece connected to a oneway non-rebreathing valve (Vitalograph, Maids Moreton, UK). Neither a nose clip nor VOC filter were used. After $5 \mathrm{~min}$ of tidal breathing the expiratory port was connected to a 2-L gas impermeable bag (Rapak, Mulgrave, Australia). The subject then inhaled to inspiratory capacity and immediately exhaled from full VC into the bag. This was connected to the CPA electronic nose within $5 \mathrm{~min}$ and the sample was drawn across the sensors at a flow rate of $120 \mathrm{~mL} \cdot \mathrm{min}^{-1}$ for $30 \mathrm{~s}$.

\section{Data analysis}

Smell prints were analysed using the Cyranose 320 inbuilt learning software. Savitzky-Golay filtering (performs a local polynomial regression (of degree $\mathrm{k}$ ) on a series of values) was utilised to process the sensor response data and baseline corrections were applied to improve signal-to-noise ratio [26]. To reduce the data from 32 individual sensors to a set of principal components, PCA was used [27]. This determined factors that captured the largest variance in the data. PCA factors were then used to perform a linear canonical discrimination analysis for the construction of a pattern recognition algorithm. This was achieved by enhancing the ratio of pooled within-class scatter to between-group distance. A cross validation value (\%) was calculated, which gave an estimate of error or, in other words, the accuracy in distinction between the smell prints from different subject groups. The Mahalanobis distance (M-distance) between group means, in units of SD, was then calculated [27]. This was used to quantify the discrimination between sample groups, providing a measure of dissimilarity between two samples. Thus, M-distance and the ability to discriminate are directly related, so that values $>3$ are indicative of a high probability of discrimination $(\mathrm{p}<0.01)$.

\section{RESULTS}

\section{Subject characteristics and lung function}

80 subjects were studied. Demographic data are shown in table 1. All healthy controls had normal spirometry. FEV1, FVC and FEV1/FVC ratio were significantly reduced in subjects with benign ARD compared with healthy control subjects $(p<0.05)$. The most significant difference was FEV1 and FVC between controls and patients with asbestosis $(100.1 \pm$ $11.1 \%$ versus $72.2 \pm 9.4 \%$ and $94.4 \pm 9.4 \%$ versus $78.9 \pm 10.4 \%$, respectively; $\mathrm{p}<0.001)$. Subjects with $\mathrm{MM}$ were unwell and unable to perform spirometry.

The MM group consisted of 20 subjects with histologically diagnosed MM. 19 subjects were International Mesothelioma Interest Group stage 2, in which the mesothelioma had spread to both layers of the pleura on one side of the body, and had enlarged to form a tumour mass on the pleural tissue around the lungs, or had started to spread into the diaphragm muscle or the lung tissue. One patient was stage 1b. Eight subjects were nonsmokers and 12 ex-smokers and all had previous asbestos exposure. The benign ARD group (pleural disease and asbestosis) comprised 18 subjects, of whom six were non-smokers and 12 ex-smokers. The healthy control group comprised 42 subjects, of which 30 were nonsmokers and 12 ex-smokers.

\section{CPA electronic nose}

Each of the 32 carbon polymer sensors in the CPA electronic nose were responsive to exhaled breath, with a recordable change in sensor resistance elicited by VOC mixtures in breath. The exhaled breath sample of each individual resulted in a unique pattern of sensor responses, which characterised their smell print (fig. 1).

\section{Mesothelioma and healthy controls}

In the training phase, 10 subjects with $\mathrm{MM}$ and 10 controls were introduced to the CPA electronic nose to create two breath recognition classes. Control subjects were recruited at the same time. Subjects were seen in random sequence and timeframe, depending upon their availability to come to the hospital for testing. Smell prints from MM patients clustered distinctly from control subjects when analysed by PCA (fig. 2). Subsequent canonical discriminant analysis showed a cross validated accuracy value of $95 \%$ (fig. 3). Canonical discriminant analysis on the data set showed an M-distance of 4.59 between the two groups, suggestive of a high probability of discrimination $(\mathrm{p}<0.01)$.

Validation on the training set was performed. The capacity of the CPA electronic nose to correctly distinguish subjects' smell prints was performed on 10 patients with MM and 32 control subjects. Exhaled breath samples were plotted against $\mathrm{MM}$ and control breath recognition classes; an example plotted " $\mathrm{X}$ " in figure 4.

TABLE 1 Subject demographics and lung function data

\begin{tabular}{|c|c|c|c|c|}
\hline & Controls & Mesothelioma & Asbestosis & Pleural disease \\
\hline Subjects & 42 & 20 & 5 & 13 \\
\hline Age yrs & $66.5 \pm 14$ & $69 \pm 10$ & $70 \pm 10.5$ & $70.9 \pm 8.2$ \\
\hline Male/female & $34 / 8$ & $18 / 2$ & $5 / 0$ & $13 / 0$ \\
\hline Nonsmoker/ex-smoker & $30 / 12$ & $8 / 12$ & $1 / 4$ & $5 / 8$ \\
\hline FEV $_{1} \%$ pred & $100.1 \pm 11.1$ & ND & $72.2 \pm 9.4^{\star \star \star}$ & $90.2 \pm 17.5^{\star}$ \\
\hline FVC \% pred & $94.4 \pm 9.4$ & ND & $78.9 \pm 10.4^{\star \star \star}$ & $82.7 \pm 18.6^{\star}$ \\
\hline FEV $_{1 / \text { FVC } \% \text { pred }}$ & $93.4 \pm 14.3$ & ND & $76.2 \pm 7.8^{\star}$ & $80.1 \pm 12.7^{\star}$ \\
\hline IMIG stage $2 /$ stage $1 \mathrm{~b}$ & NA & $19 / 1$ & NA & NA \\
\hline
\end{tabular}

Data are presented as $n$ or mean \pm SD. FEV1; forced expiratory volume in $1 \mathrm{~s}$; \% pred: \% predicted; FVC: forced vital capacity; IMIG: International Mesothelioma Interest Group; ND: not determined; NA: not applicable. *: $p<0.05$; and ***: $p<0.001$, significant differences between subjects with asbestosis or pleural plaques compared with normal controls. 


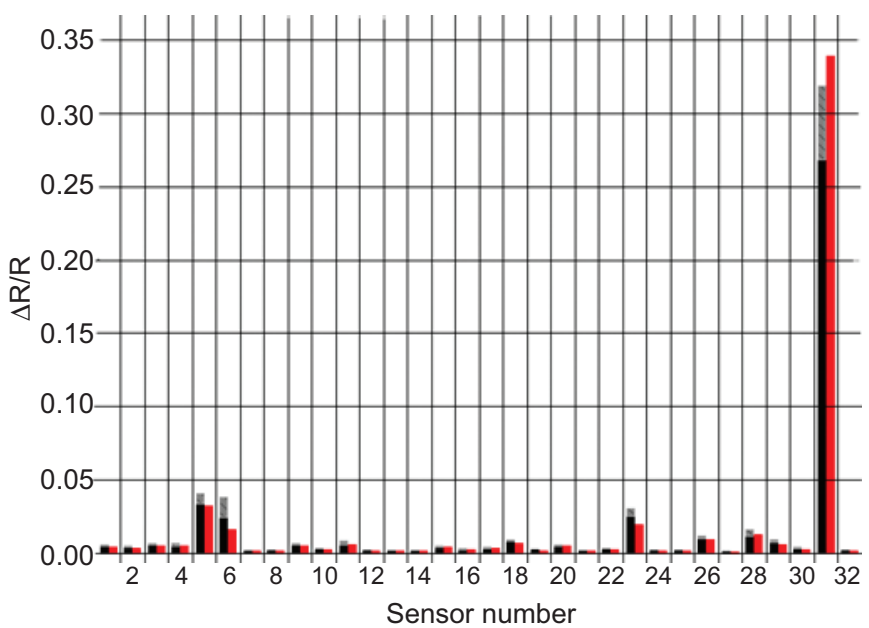

FIGURE 1. Pattern of relative differential electrical resistance $(\Delta R / R)$ of the 32 polymer sensors of the carbon polymer array electronic nose. This illustrates a smell print created by the volatile organic compound mixture in exhaled breath of a single volunteer with mesothelioma (MM). $\Delta \mathrm{R} / \mathrm{R}$ represents the change in resistance of an individual sensor. $\Delta R$ is the difference between sensor resistance recorded during "sample draw" and "baseline purge" stages of the sampling cycle and R is the resistance recorded at the end of the "baseline purge" stage (see fig. 2). The view allows comparison of a new smell print (red) with smell prints previously stored during training: the grey area is maximum exposure, and the black area is minimum exposure of an individual sensor in a training set.

Identification results were correct in 38 out of 42 subjects, with nine out of $10 \mathrm{MM}$ and 29 out of 32 controls being correctly identified (sensitivity 90\%, specificity 91\%). The incorrect

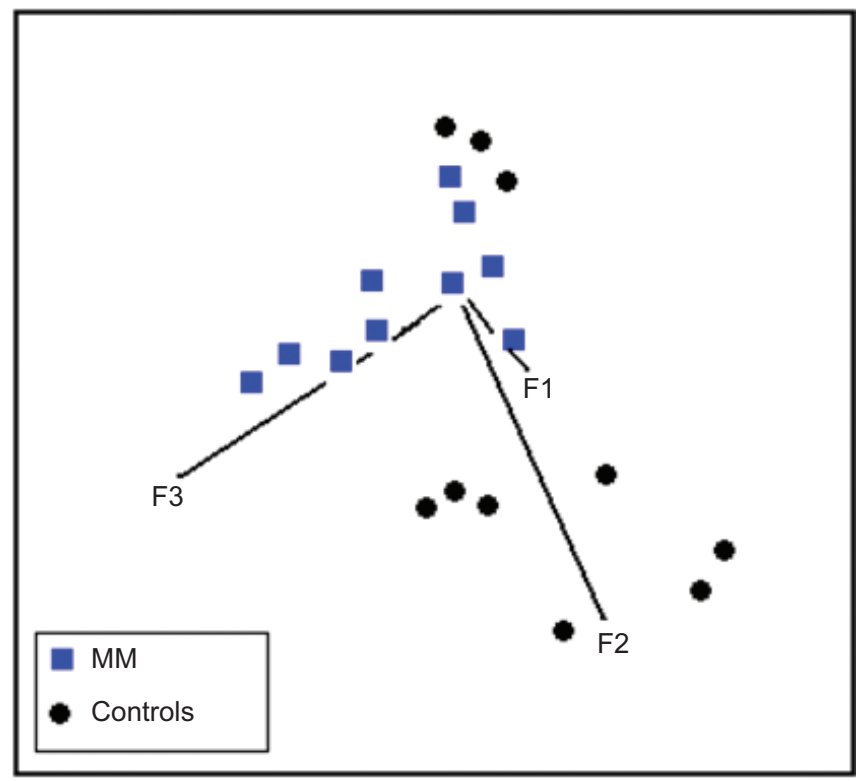

FIGURE 2. Three-dimensional principal component analysis plot classifying 10 subjects with malignant mesothelioma (MM) and 10 healthy controls. Three principal component composite factors (F1, F2 and F3) were discerned from the reduced data of the 32 sensors, maximising the discrimination of smell prints between patients with MM and healthy control subjects. The reduction to three principal components shows marked clustering of MM smell prints.

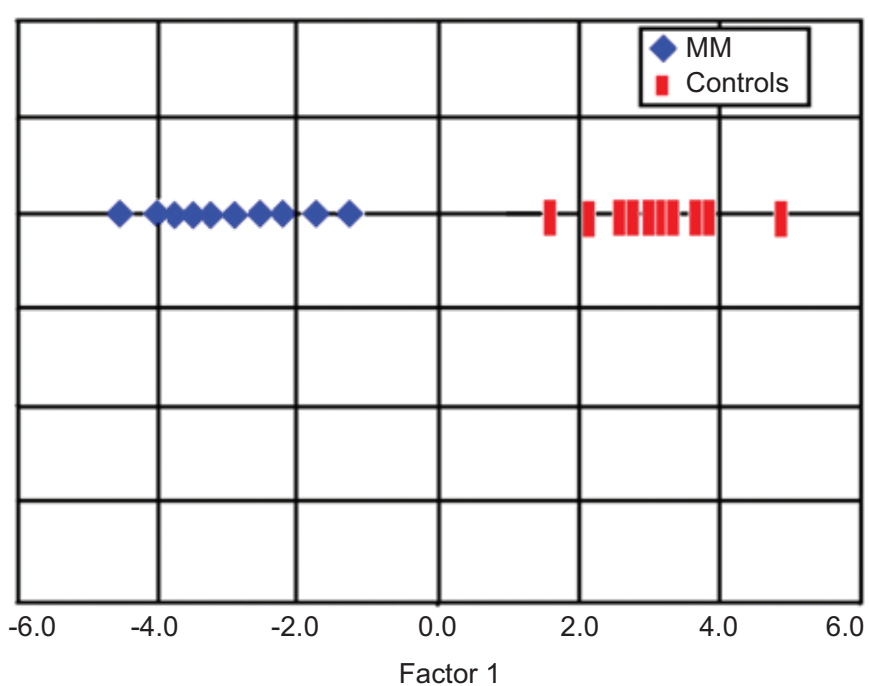

FIGURE 3. One-dimensional canonical discriminant analyses plot presenting the smell print of subjects with mesothelioma ( $\mathrm{MM})$ and healthy controls. This illustrates discrimination of subjects with MM from healthy control subjects along an arbitrary composite factor (factor 1). Factor 1 represents a single principal component, formed from the reduced data of the 32 sensors, on which canonical discriminant analysis was performed.

samples were measured again within $2 \mathrm{~h}$ and were correctly identified on the second analysis.

\section{Mesothelioma and other ARDs}

18 subjects with benign ARD (pleural disease and asbestosis) were used in a second validation phase of the MM versus healthy set. Exhaled breath samples were correctly identified

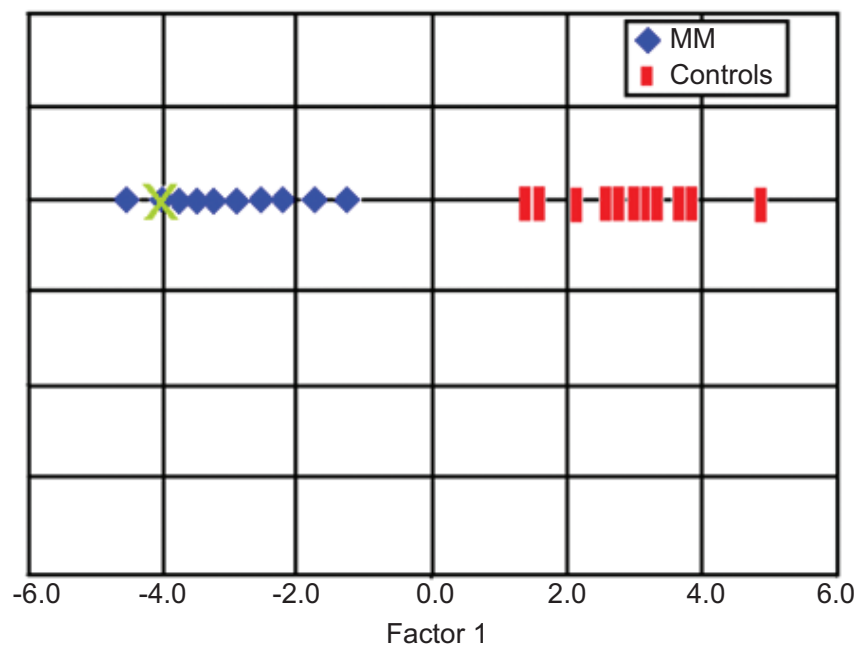

FIGURE 4. One-dimensional canonical discriminant analysis plot presenting the correct identification of a randomly introduced malignant mesothelioma (MM) subject. This illustrates discrimination of subjects with $M M(n=10)$ from healthy control subjects $(n=10)$ along an arbitrary composite factor (factor 1$)$. The smell print of a single MM subject, marked as an $\mathrm{X}$, validates the capacity of a carbon polymer array electronic nose to classify newly introduced subjects into the correct subject group, based on breath analysis alone. Factor 1 represents a single principal component on which canonical discriminant analysis was carried out. 
as healthy in 15 out of 18 subjects (specificity $83.3 \%$ ). Out of 13 patients with pleural disease alone and no asbestosis, 12 were correctly identified as healthy (92\% specificity). Three out of five patients with asbestosis smell prints were correctly identified (60\% specificity).

For smell print identification between MM, benign ARD and control subjects the CPA electronic nose had a sensitivity of $90 \%$ and specificity of $88 \%$. The positive predictive value and negative predictive values were $60 \%$ and $97.8 \%$, respectively, when compared with the gold standard of histologically proven MM.

\section{Reproducibility and intra-subject variability}

To assess reproducibility of results, the breath of five subjects was measured on three occasions. Two MM patients and three control subjects had their exhaled breath profile validated against the MM training set on three occasions over a period of 6 weeks. Breath sample validation was performed in healthy subjects at 2, 4 and 6-week time-points and the MM subjects at 1, 4 and 6-week intervals from the initial breath sample collection date. These timeframes were due to the availability of the subjects to come in for testing. This supported the hypothesis that results of the model were reproducible, with subjects being assigned to the correct class in 13 (86\%) out of 15 trials. CPA electronic nose breath prints have previously been shown to be repeatable in other studies with a kappa ranging between 0.75 and $0.91[28,29]$.

\section{DISCUSSION}

Our study has demonstrated that CPA electronic nose breath profiling allows accurate discrimination between patients with MM, healthy control subjects and subjects with benign ARDs. This is the second published report of the use of electronic nose breath profiling in $\mathrm{MM}$ and substantiates the promise of this technique as a simple, easy way for detecting malignancy which has previously been reported for lung cancer [15, 17-19]. A study by DRAGONIERI et al. [28] published very recently, while our paper was under review, also reports high levels of discrimination between patients with $\mathrm{MM}$ and those with long-term asbestos exposure using an electronic nose system, with results almost identical to those of our study. This work was performed contemporaneously with ours, substantiating the validity of both groups' results. In our study, we carefully took into account the type of ARD and a wider range of asbestos disorders, because this is known to affect exhaled breath biomarkers [5, 10], and is an important potential confounding factor for MM detection. This work exemplifies the novel use of this technology for differentiating individuals with asbestos exposure and benign conditions from those with malignant disease.

Asbestos inhalation is an important cause of disease, yet is associated with the development of malignancy in only a small percentage of occupationally exposed workers [9]. However, these patients subsequently suffer very high morbidity and mortality. Subjects with asbestos-related lung cancer are potentially curable, with reported 5-yr survival rates for stage $1 \mathrm{~A}$ lung cancer now achieving $80 \%$, compared with $<15 \%$ for stages II-IV [30, 31]. The same is not true however for MM, where survival rates have not changed significantly over the last 20 yrs $[1,3]$. However, new drugs and combined multimodality treatment offer hope for cure in selected cases. MM is usually diagnosed at a late stage and it is theoretically possible that earlier disease might be more responsive to treatment.

Our study is an early pilot approach, but has demonstrated that MM can be distinguished from normal controls and subjects with ARDs with promising accuracy. Asbestosexposed subjects have a high rate of benign pleural disorders, especially PPs. In addition, they may have interstitial pulmonary fibrosis (asbestosis), although this is becoming increasingly rare. Thus, it is important that these co-existing conditions are taken into account in order to ensure that they are not a confounder for the diagnosis of malignancy. In our study, the CPA electronic nose distinguished accurately between benign and malignant ARD, implying that the breath profile of VOCs in $\mathrm{MM}$ is very different. Although it might have been expected that MM, a pleural disease rather than an endobronchial disease, would not produce such a change in breath profile, our results are similar to a previous abstract report [28], and also to the reports of PHILLIPS and co-workers et al, [20, 32-34] whose work on lung cancer suggests that the change in VOC profile is related to a systemic alteration in metabolism which occurs with malignancy rather than due to a localised event.

Our subjects were matched for smoking status and were neveror ex-smokers, so the potentially confounding effect of smoking was not formally assessed. However, MM is not related to previous smoking habit; therefore, smoking as a confounder is not as relevant as it would be for lung cancer diagnosis. The CPA electronic nose is capable of picking up differences due to smoking status [22], but because training is specific for the VOC pattern profile relevant for a particular disease, irrelevant compounds are ignored. This implies that the effects of smoking are unlikely to have a significant confounding effect, as has been found in other studies in lung cancer and also in obstructive lung disease [15, 35, 36].

CPA electronic noses do not quantify which specific VOCs are responsible for any observed difference in exhaled breath patterns. Each of the 32 polymer sensors responds to a different fraction of the VOC mixture, based on features such as molecular mass, dipole moment and hydrogen binding capacity [35]. Significant differences in resistance are then selected by PCA. Hence, smell print pattern recognition by CPA electronic noses is purely based on a statistical approach, providing empiric evidence [36]. However, studies are underway to identify individual breath components and it is likely that these will be characterised in the near future. For example, cyclopentane and cyclohexane are likely to prove important molecules which distinguish between $\mathrm{MM}$ patients and subjects with asbestos exposure [37].

CPA electronic noses are a novel technology in medical diagnostics, and technical developments in this area are likely to increase understanding and improve methodology in the future. Because the Cyranose 320 was not developed for medical diagnostics, it has inherent limitations. It was originally designed for industrial use, assessing the identity of gaseous exposure in chemical spills, and only allows 10 samples (10 subjects) in the training set for each class. The number of MM cases we studied was small, but we were limited by the fact that the Cyranose 320 limits test validation 
to 10 subjects at a time. Ideally, larger numbers would be included in both training and validation sets to avoid potential type- 1 error. VOCs are produced both from endogenous and exogenous processes, so there is also a possibility of background environmental contamination. We did not use a VOC filter, but this is likely to improve accuracy [15, 36, 38]. However, our patients were all studied in an identical air conditioned environment typical of usual clinical practice, and there are few published data regarding optimal filter type or methods in this area. Our diagnostic accuracy was still high in these circumstances, while background contamination should, in theory, have resulted in dilution of the signal.

In our study, all subjects with MM had advanced disease, diagnosed on surgical biopsy. Thus, our study results might not be so accurate with early stages of MM. However, currently few cases of MM are detected at an early stage and histological confirmation was important as a gold standard for our study. Ideally, a prospective study design would be employed in screening an asbestos-exposed cohort, similar to work which has been performed with other biomarkers [39]. When initial case-controlled studies are translated into prospective cohort screening studies, diagnostic accuracy usually falls. However, technological developments in this area seem likely to improve both accuracy and ease of testing in this area of research. Although our data are early and require further evaluation in larger studies, our work demonstrates the potential for a convenient, hand-held noninvasive device such as a CPA electronic nose for early diagnosis of MM.

\section{SUPPORT STATEMENT}

This work was funded by the Slater \& Gordon Asbestos Research Trust and the Lesley Pockley Clinical Research Trust.

\section{STATEMENT OF INTEREST}

None declared.

\section{ACKNOWLEDGEMENTS}

The authors would like to thank the volunteers who kindly participated in this study, as well as the staff of the outpatient clinics of St Vincent's Hospital and the Prince of Wales Hospital (both Sydney, Australia).

\section{REFERENCES}

1 Park EK, Hannaford Turner K, Hyland RA, et al. Asbestos related occupational lung diseases in NSW, Australia, and potential exposure of the general population. Ind Health 2008; 46: 535-540.

2 World Health Organization. World Health Assembly Resolution 58.22 Elimination of Asbestos-Related Disease. Geneva, WHO, 2006.

3 Clements M, Berry G, Shi J, et al. Projected mesothelioma incidence in men in New South Wales, Australia. Occup Environ Med 2007; 64: 747-752.

4 Kamp DW, Weitzman SA. The molecular basis of asbestos induced lung injury. Thorax 1999; 54: 638-652.

5 Chapman EA, Thomas PS, Yates DH. Breath analysis in asbestosrelated disorders: a review of the literature and potential future applications. J Breath Res 2010; 4: 034001.

6 Hyland R, Ware S, Johnson A, et al. Incidence trends and gender differences in malignant mesothelioma in New South Wales, Australia. Scn J Work Environ Health 2007; 33: 286-292.

7 American Thoracic Society, Medical Section of the American Lung Association: the diagnosis of non-malignant diseases related to asbestos. Am Rev Respir Dis 1986; 134: 363-368.
8 Pass HI, Carbone M. Current status of screening for malignant pleural mesothelioma. Semin Thorac Cardiovasc Surg 2009; 21: 97-104.

9 Tossavainen A. Asbestos, asbestosis and cancer: the Helsinki criteria for diagnosis and attribution. Scand J Work Environ Health Perspect 1997; 23: 311-316.

10 Chow S, Campbell C, Sandrini A, et al. Exhaled breath condensate biomarkers in asbestos related lung disorders. Respir Med 2009; 103: 1091-1097.

11 Scherpereel A, Grigoriu B, Conti M, et al. Soluble mesothelinrelated peptides in the diagnosis of malignant pleural mesothelioma. Am J Respir Crit Care Med 2006; 173: 1155-1160.

12 Robinson BW, Creaney J, Lake R, et al. Soluble mesothelin-related protein - a blood test for mesothelioma. Lung Cancer 2005; 49: Suppl. 1, S109-S111.

13 Pass HI, Wali A, Tang N, et al. Soluble mesothelin-related peptide level elevation in mesothelioma serum and pleural effusions. Ann Thorac Surg 2008; 85: 265-272.

14 Creaney J, Yeoman D, Demelker Y, et al. Comparison of osteopontin, megakaryocyte potentiating factor, and mesothelin proteins as markers in the serum of patients with mesothelioma. J Thorac Oncol 2008; 3: 851-857.

15 Dragonieri S, Annema JT, Schot R, et al. An electronic nose in the discrimination of patients with non-small cell lung cancer and COPD. Lung Cancer 2009; 64: 166-170.

16 Horvath I, Lázár Z, Gyulai N, et al. Exhaled biomarkers in lung cancer. Eur Respir J 2009; 34: 261-275.

17 Phillips M, Altorki N, Austin JHM. Detection of lung cancer using weighted digitial analysis of breath biomarkers. Clin Chim Acta 2008; 393: 76-84.

18 Wehinger A, Schmid A, Mechtcheriakov S, et al. Lung cancer detection by proton transfer reaction mass-spectrometric analysis of human breath gas. Int J Mass Spec 2007; 265: 49-59.

19 Mazzone PJ, Hammel J, Dweik RA, et al. Lung cancer diagnosis by the analysis of exhaled breath with a colorimetric sensor array. Thorax 2007; 62: 565-568.

20 Phillips M, Altorki N, Austin JHM, et al. Prediction of lung cancer using volatile biomarkers in breath. Cancer Biomarkers 2007; 3: 95-109.

21 McCulloch M, Jezierski T, Broffman M, et al. Diagnostic accuracy of canine scent detection in early- and late-stage lung and breast cancers. Intergr Cancer Ther 2006; 5: 30-39.

22 Cheng ZJ, Warwick G, Yates DH, et al. An electronic nose in the discrimination of breath from smokers and non-smokers: a model for toxin exposure. J Breath Res 2009; 3: 36-41.

23 British Thoracic Society Standards of Care Committee. BTS statement on malignant mesothelioma in the UK, 2007. Thorax 2007; 62: Suppl. 2, ii1-ii19.

24 Miller MR, Hankinson J, Brusasco V, et al. Standardisation of spirometry. Eur Respir J 2005; 26: 319-338.

25 Cotes JE, Chinn DJ, Quanjer PH. Standardization of the measurement of transfer factor (diffusing capacity). Report Working Party Standardization of Lung Function Tests European Community for Steel and Coal. Eur Respir J 1993; 6: Suppl. 16, 41-52.

26 Savitsky A, Golay MJE. Smoothing and differentiation of data by simplified least square procedures. Anal Chem 1964; 36: 1627-1639.

27 Wold S, Ebensen K, Geladi P. Principal component analysis. Chemom Intell Lab Syst 1987; 2: 37-52.

28 Dragonieri S, van der Schee MP, Massaro T, et al. An electronic nose distinguishes exhaled breath of patients with malignant pleural mesothelioma from controls. Lung Cancer 2012; 75: 326-331.

29 Fens N, Zwinderman AH, van der Schee MP, et al. Exhaled breath profiling enables discrimination of chronic obstructive pulmonary disease and asthma. Am J Respir Crit Care Med 2009; 180: 1076-1082.

30 Ettinger DS, Akerley W, Bepler G, et al. Non-small cell lung cancer. J Natl Compr Canc Netw 2010; 8: 740-801. 
31 Agarwala M, Brahmandaya G, Chmielewskib GW, et al. Age, tumor size, type of surgery, and gender predict survival in early stage (stage I and II) non-small cell lung cancer after surgical resection. Lung Cancer 2010; 68: 398-402.

32 Phillips M, Cantaneo R, Cummin A, et al. Detection of lung cancer with volatile markers in the breath. Chest 2003; 123: 2115-2123.

33 Phillips M, Greenberg J, Awad J. Metabolic and environmental origins of volatile organic compounds in breath. J Clin Pathol 1994; 47: 1052-1053.

34 Phillips M, Gleeson K, Hughes J, et al. Volatile organic compounds in breath as markers of lung cancer: a cross-sectional study. Lancet 1999; 535: 1930-1933.

35 Machado R, Laskowski D, Deffenderfer O, et al. Detection of lung cancer by sensor array analysese of exhaled breath. Am J Respir Crit Care Med 2005; 171: 1286-1291.
36 Dragonieri S, Schot R, Mertens BJA, et al. An electronic nose in the discrimination of patients with asthma and controls. J Allergy Clin Immunol 2007; 120: 856-862.

37 de Gennaro G, Dragonieri S, Longobardi F, et al. Chemical characterization of exhaled breath to differentiate between patients with malignant plueral mesothelioma from subjects with similar professional asbestos exposure. Anal Bioanal Chem 2010; 398: 3043-3050.

38 Moser B, Bodrogi F, Eibl G, et al. Mass spectrometric profile of exhaled breath: field study by PTR-MS. Respir Physiol Neurobiol 2005; 145: 295-300.

39 Park EK, Sandrini A, Yates DH, et al. Soluble mesothelinrelated protein in an asbestos-exposed population: the dust diseases board cohort study. Am J Respir Crit Care Med 2008; 178: 832-837. 